\title{
A Class of Linear Boundary Value Problem for $k$-regular Functions in Clifford Analysis
}

\author{
Yan Zhang ${ }^{1}$ \\ ${ }^{1}$ School of Mathematics and Information Science, Beifang University of Nationalities, Yinchuan 750021, P. R. China. \\ E-mail: yzhangbun@163.com
}

Received: April 16, 2016 Accepted: April 24, 2016 Online Published: May 18, 2016

doi:10.5539/jmr.v8n3p57

URL: http://dx.doi.org/10.5539/jmr.v8n3p57

\section{Abstract}

In this paper, we introduce the linear boundary value problem for $k$-regular function, and give an unique solution for this problem by integral equation method and fixed-point theorem.

Keywords: $k$-regular function, Clifford analysis

\section{Introduction}

The boundary value problem is one of the important aspects in Clifford analysis. This problem on bounded domains has seen great achievements. [Wen, 1991; Huang, 1996; Zhang et al., 2001] have discussed Riemann-Hilbert boundary value problems of regular function on bounded domains. [Li, 2007] characterized boundary value problems of $k$ - regular functions. In this paper, we introduce the linear boundary value problem of $k$-regular function, and give an unique solution to this problem by integral equation method and fixed-point theorem.

Let $n$ be a positive integer, and $\left\{e_{0}, e_{1}, \cdots, e_{n}\right\}$ be basis for the Euclidean space $\mathbb{R}^{n+1}$. We denote by $\mathcal{A}$ the $2^{n}$ dimensional real Clifford algebra, which is generated by $\mathbb{R}^{n+1}$; denote the basis of $\mathcal{A}$ by $e_{A}=e_{\alpha_{1}, \alpha_{2}, \cdots, \alpha_{h}}, A=\left\{\alpha_{1}, \alpha_{2}, \cdots, \alpha_{h}\right\} \subseteq$ $\{1,2, \cdots, n\}, 1 \leq \alpha_{1}<\alpha_{2}<\cdots<\alpha_{h} \leq n$. In particular, if $A=\emptyset, e_{\phi}=e_{0}$. So, for an arbitrary $u \in \mathcal{A}$, we have $u=\sum_{A} u_{A} e_{A}$ with $u_{A} \in \mathbb{R}$. In $\mathcal{A}$, we have

$$
e_{i}^{2}=-1, e_{i} e_{j}=-e_{j} e_{i} \text { for } i \neq j, i, j=1,2, \cdots, n,
$$

that is so-called combinative and incommutable multiplication rule of Clifford algebra. For $u \in \mathcal{A}$, we write $u^{*}=$ $\sum_{A}(-1)^{\frac{|A|(|A|-1)}{2}} u_{A} e_{A}, u^{\prime}=\sum_{A}(-1)^{\frac{|A|}{2}} u_{A} e_{A}$ and $|u|$ for its module, where $|A|$ is the cardinality of the index set $A$. Define $|u|^{2}=\sum_{A}\left|u_{A}\right|^{2} ; \bar{u}$ its conjugate with $\bar{u}=\left(u^{*}\right)^{\prime}$, where $u^{*}=\sum_{A}(-1)^{\frac{|A|(|A|-1)}{2}} u_{A} e_{A}$, and $u^{\prime}=\sum_{A}(-1)^{|A|} u_{A} e_{A}$. For $u$, ve $\mathcal{A}$, we have

$$
|u+v| \leq|u|+|v|,|u v| \leq 2^{n}|u||v| .
$$

Let $D$ be a region in $\mathbb{R}^{n+1}$. For a differentiable function $f: D \rightarrow \mathcal{A}$ with $f(x)=\sum_{A} f_{A}(x) e_{A}$, we say $f$ is a regular function if

$$
\bar{\partial} f=\sum_{i=0}^{n} e_{i} \frac{\partial f}{\partial x_{i}}=\sum_{i=0}^{n} \sum_{A} e_{i} e_{A} \frac{\partial f_{A}}{\partial x_{i}}=0,
$$

and a $k$-regular function if $\bar{\partial}^{k} f=0$, where the operator $\bar{\partial}=\sum_{j=0}^{n} \frac{\partial}{\partial_{x_{j}}} e_{j}$. Let $\Omega \subset \mathbb{R}^{n+1}$ be an unbounded domain with smooth oriented Liapunove boundary $\partial \Omega$, and $\Omega^{c}$, the complementary set of $\Omega$ containing a non-empty open set. We denote the bounded Hölder continuous function on $\partial \Omega$ in order of $\beta(0<\beta<1)$ by $H(\partial \Omega, \beta)$. For $f \in H(\partial \Omega, \beta)$, we define its norm by

$$
\|f\|_{\beta}=\sup _{t \in \partial \Omega}|f(t)|+\sup _{t_{1} \neq t_{2}} \frac{\left|f\left(t_{1}\right)-f\left(t_{2}\right)\right|}{\left|t_{1}-t_{2}\right|} .
$$

Then $H\left(\partial \Omega,\|\cdot\|_{\beta}\right)$ is a Banach space. And for $f, g \in H\left(\partial \Omega,\|\cdot\|_{\beta}\right)$, we have

$$
\|f+g\|_{\beta} \leq\|f\|_{\beta}+\|g\|_{\beta},\|f g\|_{\beta} \leq 2^{n}\|f\|_{\beta}\|g\|_{\beta} .
$$

\section{Main Result}

In what follows, we denote by $\Omega$ a non-empty connected open set in $\mathbb{R}^{n+1}$ with smooth oriented Liapunove boundary $\partial \Omega$, and by $w_{n}$ the area of unit ball in $\mathbb{R}^{n+1}$. We first give the linear boundary value problem for $k$-regular function.

Definition 2.1. Let $A(t), B(t), g_{l}(t) \in H(\partial \Omega, \beta), 1 \leq l \leq k$. Write $\Omega^{+}=\Omega, \Omega^{-}=\mathbb{R}^{n+1} \backslash \bar{\Omega}$ with $\bar{\Omega}=\Omega \cup \partial \Omega$. If there exists some function $\phi$ such that 
1) $\phi$ is a $k$-regular function on $\Omega^{ \pm}$;

2)

$$
\left\{\begin{array}{l}
\phi^{+}(t) A(t)+\phi^{-}(t) B(t)=g_{1}(t) \\
\bar{\partial} \phi^{+}(t) A(t)+\bar{\partial} \phi^{-}(t) B(t)=g_{2}(t) \\
\quad \vdots \\
\bar{\partial}^{k-1} \phi^{+}(t) A(t)+\bar{\partial}^{k-1} \phi^{-}(t) B(t)=g_{k}(t)
\end{array}\right.
$$

Then we say $\phi$ is a solution to the linear boundary problem. And this problem is also called linear boundary problem for $k$-regular function.

The following lemmas are borrowed from [Li, 2007]:

Lemma 2.1. Let $f(x)$ be a $k$-regular function on $\Omega$. Then we have

$$
f(x)=\sum_{m=0}^{k-1} \frac{1}{m !} x_{0}^{m} f_{m}(x),
$$

where $f_{m}, m=0,1, \cdots, k-1$ are regular functions defined on $\Omega$.

Lemma 2.2 Here we give Plemelj equation for regular function:

$$
\phi_{m}^{ \pm}= \pm \frac{1}{2} \varphi_{m}+\frac{1}{w_{n}} \int_{\partial \Omega} \frac{\overline{\tau-x}}{|\tau-x|^{n+1}} m(\tau) \varphi_{m}(\tau) d_{s_{\tau}} .
$$

where $m(u)$ is the unit vector in $\partial \Omega$ 's normal direction, and $\varphi_{j} \in H(\partial \Omega, \beta), j=0,1, \cdots, k-1$. Then $\phi$ is a regular function on $\mathbb{R}^{n+1} \backslash \partial \Omega$.

The following lemma is borrowed from [Xu et al., 2008]

Lemma 2.3. Let $\phi \in H(\partial \Omega, \beta)$. Define a operator $K$ on $H(\partial \Omega, \beta)$ by

$$
(K \phi)(x)=\frac{1}{w_{n}} \int_{\partial \Omega} \frac{\overline{\tau-x}}{|\tau-x|^{n}} m(\tau) \phi(\tau) d_{s_{\tau}}
$$

for $x \in \partial \Omega$. Then there exists some $C>0$ such that $\|K \cdot\| \leq C\|\cdot\|$ on $H(\partial \Omega, \beta)$.

Theorem 2.1. Let $A(t), B(t), g_{l}(t),(1 \leq l \leq k) \in H(\partial \Omega, \beta)$. If

$$
\begin{aligned}
& \zeta=2^{n}\left[\left(\frac{1}{2}+C\right)(\|A+B\|)+\|1-B\|\right] \in(0,1), \\
& \left\|g_{m+1}^{\prime}\right\|_{\beta} \leq M(1-\zeta),
\end{aligned}
$$

where $C$ is in Lemma 2.3, then the solution of the $m$-th equation in (1) is given by

$$
\phi(x)=\sum_{m=0}^{k-1} \frac{1}{m !} x_{1}^{m} \phi_{m}(x)
$$

with

$$
\phi_{m}=\frac{1}{w_{n}} \int_{\partial \Omega} \frac{\overline{\tau-x}}{|\tau-x|^{n}} m(\tau) \varphi_{m}(\tau) d_{s_{\tau}}
$$

for $m=0,2, \cdots, k-1$.

Proof. Substituting (2) into (1), we have

$$
T\left(\begin{array}{c}
\phi_{0}^{+} \\
\vdots \\
\phi_{k-2}^{+} \\
\phi_{k-1}^{+}
\end{array}\right) A+T\left(\begin{array}{c}
\phi_{0}^{-} \\
\vdots \\
\phi_{k-2}^{-} \\
\phi_{k-1}^{-}
\end{array}\right) B=\left(\begin{array}{c}
g_{1} \\
\vdots \\
g_{k-1} \\
g_{k},
\end{array}\right)
$$


where

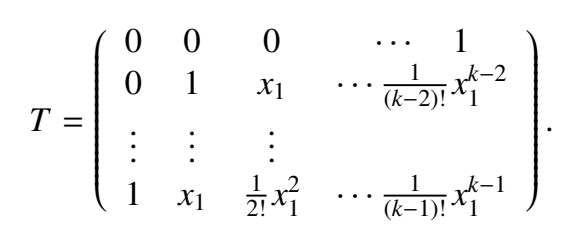

We rewrite (6) as

$$
\left(\begin{array}{cc}
\phi_{0}^{+} & A \\
\vdots & \vdots \\
\phi_{k-2}^{+} & A \\
\phi_{k-1}^{+} & A
\end{array}\right)+\left(\begin{array}{cc}
\phi_{0}^{+} & B \\
\vdots & \vdots \\
\phi_{k-2}^{+} & B \\
\phi_{k-1}^{+} & B
\end{array}\right)=\left(\begin{array}{c}
g_{1}^{\prime} \\
\vdots \\
g_{k-1}^{\prime} \\
g_{k}^{\prime}
\end{array}\right) \text { with }\left(\begin{array}{c}
g_{1}^{\prime} \\
\vdots \\
g_{k-1}^{\prime} \\
g_{k}^{\prime}
\end{array}\right)=T^{-1}\left(\begin{array}{c}
g_{1} \\
\vdots \\
g_{k-1} \\
g_{k}
\end{array}\right)
$$

which is equivalent to

$$
\left\{\begin{array}{c}
\phi_{0}^{+} A+\phi_{0}^{-} B=g_{1}^{\prime} \\
\phi_{1}^{+} A+\phi_{1}^{-} B=g_{2}^{\prime} \\
\vdots \\
\phi_{k-1}^{+} A+\phi_{k-1}^{-} B=g_{k}
\end{array}\right.
$$

herein $\phi_{m}$ is a regular function given by

$$
\phi_{m}=\frac{1}{w_{n}} \int_{\partial \Omega} \frac{\overline{\tau-x}}{|\tau-x|^{n}} m(\tau) \varphi_{m}(\tau) d_{s_{\tau}}
$$

for $m=0, \cdots, k-1$. Next, to finish the proof, we only need to prove that $\phi_{m}(0 \leq m \leq k-1)$ given by (8) are solutions to (7). By substituting (3) into (7), we have

$$
\left(\frac{1}{2} \varphi_{m}+K \varphi_{m}\right) A+\left(-\frac{1}{2}\right) \varphi_{m}+K \varphi_{m} B=g_{m+1}^{\prime} \quad m=0, \cdots, k-1 .
$$

Write

$$
L \phi_{m}=\left(\frac{1}{2} \varphi_{m}+K \varphi_{m}\right)(A+B)+\varphi_{m}(1-B)-g_{m+1}^{\prime},
$$

then (9) can be rewritten as $L \phi_{m}=\phi_{m}$. Let $T=\left\{\varphi \mid \varphi \in H(\partial \Omega, \beta),\|\varphi\|_{\beta} \leq M\right\}$. Then $T$ is a closed subspace of $H(\partial \Omega, \beta)$. Since

$$
\begin{aligned}
\left\|L \varphi_{m}\right\|_{\beta} & =\left\|\left(\frac{1}{2} \phi_{m}+K \phi_{m}\right)(A+B)+(1-B) \phi_{m}-g_{m+1}^{\prime}\right\| \\
& \leq 2^{n}\left[\left(\frac{1}{2}+C\right)(\|A+B\|)+\|1-B\|\right]\left\|\phi_{m}\right\|+\left\|g_{m+1}^{\prime}\right\| \\
& \leq \zeta\left\|\phi_{m}\right\|+\left\|g_{m+1}^{\prime}\right\| \\
& \leq M,
\end{aligned}
$$

$F$ is a map on $T$. For $\phi_{m}^{\prime}, \phi_{m}^{\prime \prime} \in T$, we have

$$
\left\|L \phi_{m}^{\prime}-L \phi_{m}^{\prime \prime}\right\| \leq \zeta\left\|\phi_{m}^{\prime}-\phi_{m}^{\prime \prime}\right\|_{\beta}
$$

with $0<\zeta<1$, and thus $L$ is a compression map on $T$. So, there is an unique fixed $\varphi_{m}$ such that $L \varphi_{m}=\varphi_{m}$ by fixed point theorem, which implies that

$$
\phi_{m}=\frac{1}{w_{n}} \int_{\partial \Omega} \frac{\overline{\tau-x}}{|\tau-x|^{n}} m(\tau) \varphi_{m}(\tau) d_{s_{\tau}}
$$

is unique solution for the $m$-th equation in (7). This gives the proof.

\section{Acknowledgements}

This work was supported by National Natural Science Foundation of China (Grant No. 11501010). The author thanks the referees for their valuable suggestions, which greatly improve the readability of the article. 


\section{References}

Brackx, F., Delange, R., \& Sommen, F. (1982). Clifford Analysis, London:Pitman Advanced Publishing Program.

Huang, S. (1996). A nonlinear boundary problem with Haseman shift in Cliford analysis. Journal of Systems Science and Mathematical Science (Chinese edition), 16, 60-64.

Huang, S. (1996). The nonlinear boundary problem of bi-regular functions in Clifford analysis. Science China, 36A, $1152-1164$.

Li, J. Y. (2007). The property and Riemann boundary problem of $k$-regular function in Clifford analysis. Journal of Sichuan Normal University (Natural Sci. edition), 30, 430-433.

Li, J. Y., \& Yang, P. W. (2006). The nonlinear boundary problem of a class of generalized regular functions. Journal of Sichuan Normal University (Natural Sci. edition), 29, 30-33.

Wen, G. C. (1991). Clifford analysis and elliptic system, hyperbolic systems of first order equations, London: World Scientific.

Xu, N., \& Qiao, Y. Y. (2008). The boundary problem of $k$-regular functions on unbounded domain in Clifford analysis. Acta Mathematica Scientia, 28A, 846-855.

Xu, Z. Y. (1987). The Riemann boundary problem of regular function on Clifford algebra. Chinese Science Bulletin, 32, 476-477.

Yang, L., \& Zeng, C. Y. (2008). The Riemann boundary problem of $k$-regular functions on unbounded domain in Clifford analysis. Journal of Sichuan Normal University (Natural Sci. edition), 31, 693-697.

Yang, P. W. (1998). The regular functions in complex Clifford analysis. Journal of Sichuan Normal University (Natural Sci. edition), 19, 67-68.

Zeng, W., \& Yang, P. W. (2007). The irregular Riemann boundary problem of $k$-regular function. Journal of Sichuan Normal University (Natural Sci. edition), 30, 291-294.

Zhang, W. Q., \& Yang, P. W. (2006). A conjugate value boundary problem of $k$-regular function. Journal of Sichuan Normal University (Natural Sci. edition), 29, 249-251.

Zhang, Z. X., \& Du, J. Y. (2001). Some Riemann boundary problems and singular integral equation in Clifford analysis. Chinese Annals of Mathematics, 22A, 421-426.

Zhao, Z. (1995). The property and Hilbert boundary problem of bi-analytic functions. Journal of Beijing Normal University (Natural Sci. edition), 31, 175-179.

\section{Copyrights}

Copyright for this article is retained by the author(s), with first publication rights granted to the journal.

This is an open-access article distributed under the terms and conditions of the Creative Commons Attribution license (http://creativecommons.org/licenses/by/3.0/). 